\title{
Requirements Justification to Digital Signal Synthesizers for Various Types of Radar Systems
}

\author{
Dmitriy S. Viktorov*, \\ Ekaterina V. Plastinina and Elena V. Samovolina \\ Military Academy of Aero-Space Defence \\ named after the Marshal of Soviet Union G.K. Zhukov \\ Tver, Russian Federation
}

Received 01.09.2020, received in revised form 10.09.2020, accepted 15.09.2020

\begin{abstract}
The paper justifies the requirements for the level of distortion of radar stations with pulse and quasi-continuous radiation, built on the basis of digital signal synthesizers of four types: digital synthesizers of voltage counts and digital synthesizers of phase counts with uniform sampling, digital synthesizers of voltage counts and digital synthesizers of phase counts with uneven sampling. When building a radar master device, the question arises about choosing the type of digital signal synthesizer. The main initial criteria are the maximum operating range of the digital synthesizer and the level of in-band distortion. When choosing the type of digital signal synthesizer, you have to take into account a large number of factors, the main of which are the complexity of the execution of the digital readout shaper, the possibility of implementing a digital readout shaper with the required speed and number of digits $[1,2]$. When making requirements for the total level of distortion, the criterion of acceptable reduction of the probability of correct detection in comparison with its potential value for a fixed probability of false alarm is used. Based on this criterion, in pulse radars, the maximum relative RMS value of the distortion of the intercorrelation function of a signal with angular modulation generated by a digital synthesizer should not exceed $D_{\delta x} \leq-(51 \ldots 67) \mathrm{dB}$. In a radar with quasi-continuous radiation, the maximum relative mean-square value of the distortion of the autocorrelation function of the signal with angular modulation should not exceed $D_{\delta} \leq-(80 \ldots 120) \mathrm{dB}$. The number of bits of phase quantization, voltage and time delay compensation in digital signal synthesizers depends not only on the maximum relative RMS value of the distortion of the intercorrelation function, but also on the number of samples of the signal with angular modulation. Therefore, initially you need to select the reference frequency of the digital signal synthesizer, setting the type of modulation and the effective spectrum width of the signal with angular modulation based on the tactical and technical characteristics radar.
\end{abstract}

Keywords: digital signal synthesizers, phase and voltage quantization, sample rate.

Citation: Viktorov D.S., Plastinina E.V., Samovolina E.V. Requirements justification to digital signal synthesizers for various types of radar systems, J. Sib. Fed. Univ. Eng. \& Technol., 2020, 13(7), 818-828. DOI: 10.17516/1999-494X-0268

(C) Siberian Federal University. All rights reserved

This work is licensed under a Creative Commons Attribution-Non Commercial 4.0 International License (CC BY-NC 4.0).

* Corresponding author E-mail address: viktorov.dmitry@yandex.ru 


\title{
Обоснование требований
}

\author{
к цифровым синтезаторам сигналов \\ для различных типов РЛС
}

\author{
Д.С. Викторов, Е.В. Пластинина, Е.В. Самоволина \\ Военная академия воздушно-космической оборонь \\ им. Маршала Советского Союза Г.К. Жукова \\ Российская Федерация, Тверь
}

\begin{abstract}
Аннотация. Обоснованы требования к уровню искажений радиолокационных станций с импульсным и квазинепрерывным излучениями, построенных на основе цифровых синтезаторов сигналов четырех типов: цифровых синтезаторов отсчетов напряжения и цифровых синтезаторов отсчетов фазы с равномерной дискретизацией, цифровых синтезаторов отсчетов напряжения и цифровых синтезаторов отсчетов фазы с неравномерной дискретизацией. При построении задающего устройства РЛС возникает вопрос о выборе типа цифрового синтезатора сигналов. Основными исходными критериями при этом являются максимальный рабочий диапазон цифрового синтезатора и уровень внутриполосных искажений. При выборе типа цифрового синтезатора сигналов необходимо учитывать большое количество факторов, основные из которых сложность исполнения формирователя цифровых отсчетов, возможность реализации формирователя цифровых отсчетов с требуемым быстродействием и количеством разрядов $[1,2]$. При предъявлении требований к суммарному уровню искажений используют критерий допустимого снижения вероятности правильного обнаружения по сравнению с ее потенциальным значением при фиксированной вероятности ложной тревоги. Исходя из данного критерия в импульсных РЛС максимальное относительное среднеквадратическое значение искажений взаимокорреляционной функции сигнала с угловой модуляцией, формируемого цифровым синтезатором, не должно превышать $\mathrm{D} \delta \mathrm{x} \leq-(51 . .67)$ дБ. В РЛС с квазинепрерывным излучением максимальное относительное среднеквадратическое значение искажений автокорреляционной функции сигнала с угловой модуляцией не должно превышать $\mathrm{D} \delta \leq-(80 . .120)$ дБ. Количество разрядов квантования фазы, напряжения и компенсации временной задержки в цифровых синтезаторах сигналов зависит не только от максимального относительного среднеквадратического значения искажений взаимокорреляционной функции, но и от количества отсчетов сигнала с угловой модуляцией. Поэтому первоначально необходимо выбрать эталонную частоту цифрового синтезатора сигналов, задаваясь видом модуляции и эффективной шириной спектра сигнала с угловой модуляцией исходя из ТТХ РЛС.
\end{abstract}

Ключевые слова: цифровые синтезаторы сигналов, квантование фазы и напряжения, частота дискретизации.

Цитирование: Викторов, Д.С. Обоснование требований к цифровым синтезаторам сигналов для различных типов РЛС / Д.С. Викторов, Е.В. Пластинина, Е.В. Самоволина // Журн. Сиб. федер. ун-та. Техника и технологии, 2020. 13(7). C. 818-828. DOI: $10.17516 / 1999-494 X-0268$

В [3-10] показано, что квантование фазы и напряжения, дискретизация и интерполяция сигналов в цифровых синтезаторах приводят к появлению модуляционных и внутриполосных искажений спектров формируемых сигналов с угловой модуляцией (УМ) и, следовательно, их корреляционных функций (уменьшения амплитуды, временного смещения и расширения основного лепестка, росту боковых лепестков сжатого импульса). В конечном счете это приводит к снижению потенциальных тактико-технических характеристик (ТTХ) РЛС и является основным фактором, ограничивающим использование в них цифровых синтезаторов сигналов (ЦСС).

$$
-819-
$$


Поэтому разработка требований, предъявляемых к ЦСС, для различных типов РЛС является актуальной задачей.

\section{Требования к уровню искажений в РЛС с импульсным и квазинепрерывным излучениями}

В РЛС с корреляционно-фильтровым методом обработки радиолокационных сигналов с УМ можно выделить четыре основных источника искажений $(I=4)$ : ЦСС сигналов с УМ, усилитель мощности передающего канала, усилительный тракт приемного канала и гетеродин $[11,12]$. Указанные устройства имеют независимое конструктивное исполнение, поэтому обусловленные ими искажения в первом приближении можно считать некоррелированными, а их вклады в суммарные искажения сигналов на выходе схем обработки примерно одинаковыми. Тогда суммарная спектральная плотность мощности шумов, приведенных к входу приемного устройства, от $I$ некоррелированных источников искажений равна

$$
N=\sum_{i=1}^{I} N_{i},
$$

где $N_{i}$ - спектральная плотность мощности $i$-го источника шума.

В этом случае допустимая спектральная плотность мощности искажений в ЦСС с УМ не должна превышать $N_{\text {цС }}<N / I$.

Искажения, возникающие при формировании сигналов с УМ в цифровых синтезаторах, в свою очередь, можно разделить на регулярные и случайные. Регулярные искажения возникают при квантовании и дискретизации формируемого в цифровом синтезаторе сигнала. Эти искажения для каждого вида формируемого сигнала с УМ повторяются от импульса к импульсу. Случайные искажения обусловлены мультипликативным (модуляционным) и аддитивным воздействиями шумов и нестабильностей источников эталонов, источников питания, электронных приборов и т.д. [13, 14]. В первом приближении можно считать, что спектральная плотность мощности регулярных $N_{\mathrm{P}}$ и случайных $N_{\mathrm{C}}$ искажений имеет равномерный уровень в полосе рабочего диапазона ЦСС и равна между собой. Тогда,

$$
N_{Ц \mathrm{C}} \leq N_{\mathrm{P}}+N_{\mathrm{C}}=2 N_{\mathrm{P}}
$$

С учетом сказанного выше, допустимая спектральная плотность мощности регулярных искажений в ЦСС с УМ не должна превышать

$$
N_{\mathrm{P}} \leq N /(2 I)
$$

На практике при предъявлении требований к суммарному уровню указанных искажений исходят из допустимого ухудшения основных ТТХ РЛС. Для большинства классов РЛС одним из важнейших исходных критериев является допустимое снижение вероятности правильного обнаружения по сравнению с ее потенциальным значением $\Delta P_{D}=P_{D}-P_{D}{ }^{\prime}$ при фиксированной вероятности ложной тревоги $P_{F}[15]$.

Для обоснования требований к допустимому уровню искажений сигналов с УМ, формируемых ЦСС, применительно к импульсным РЛС рассмотрим случай обнаружения сигнала $X_{1}(t)$, отраженного от малоразмерной цели 1 , на фоне шумов $n(t)$ и искажений $\delta X_{2}(t)$ сигнала $X_{2}(t)$, от-

$$
-820-
$$




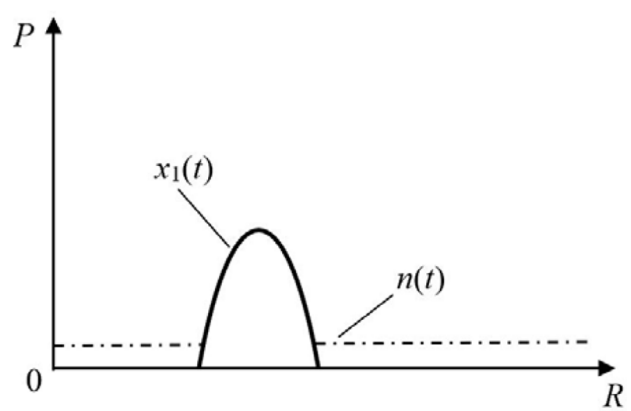

a)

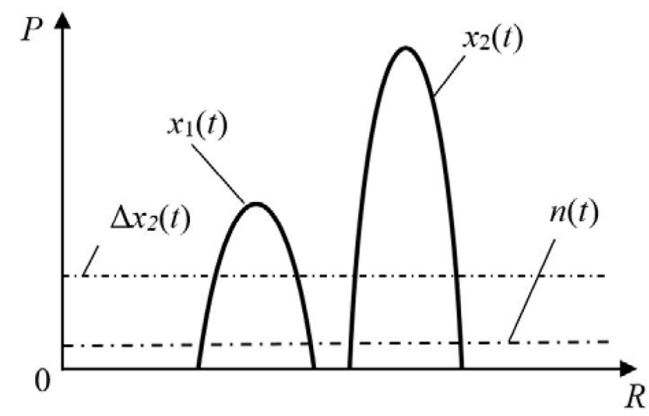

б)

Рис. 1

Fig. 1

раженного от близко расположенной к цели 1 крупноразмерной цели 2 (рис. 1). При отсутствии цели 2 (рис. 1а) параметр обнаружения цели 1 определяется значением

$$
q_{1}=\sqrt{\frac{2 E_{1}}{N_{0}}}
$$

где: $\mathrm{E}_{1}$ - энергия сигнала, отраженного от цели $1 ; N_{0}$ - спектральная плотность мощности шума $n(t)$.

При наличии цели 2 (рис. 1б) параметр обнаружения цели 1 на фоне шумов $n(t)$ и искажений $\delta X_{2}(t)$ определяется значением

$$
q_{2}=\sqrt{\frac{2 E_{1}}{N_{0}+N}}
$$

где $N$ - спектральная плотность мощности суммарных искажений в $X_{2}(t)$ сигнала $X_{2}(t)$.

Тогда спектральную плотность мощности можно определить в виде

$$
N=2 \frac{q_{1}^{2}-q_{2}^{2}}{q_{1}^{2} q_{1}^{1}} E_{1} .
$$

Разделив левую и правую части равенства на значение $\mathrm{E}_{2}$ и учитывая, что отношение $\mathrm{E}_{1} / \mathrm{E}_{2}$ пропорционально отношению эффективных площадей рассеяния этих целей $S_{1} / S_{2}$, получим

$$
\frac{N}{E_{2}}=2 \frac{q_{1}^{2}-q_{2}^{2}}{q_{1}^{2} q_{1}^{1}} \frac{S_{1}}{S_{2}} \text { или } \frac{N N_{\mathrm{p}}}{E_{2}} \leq \frac{1}{I} \frac{q_{1}^{2}-q_{2}^{2}}{q_{1}^{2} q_{1}^{1}} \frac{S_{1}}{S_{2}} .
$$

В свою очередь, отношение $N_{\mathrm{p}} / E_{2}$ пропорционально максимальному относительному среднеквадратическому значению искажений взаимокорреляционной функции (ВКФ) $\sigma_{\Delta \chi}^{2}$ сигналов с УМ, формируемых в ЦСС. Поэтому требование к допустимому значению $\sigma_{\Delta \chi}^{2}$ можно определить в виде:

$$
\sigma_{\Delta \chi}^{2} \leq \frac{1}{I} \frac{q_{1}^{2}-q_{2}^{2}}{q_{1}^{2} q_{1}^{1}} \frac{S_{1}}{S_{2}}
$$


Задаваясь допустимым уменьшением $\Delta P_{d}=0.05 \ldots 0.1$ при $P_{d}=0.8 \ldots 0.9, P_{F}=10^{-8}, S_{1} / S_{2}=0.002$ и $I=4$, получим $\sigma_{\Delta \chi}^{2} \leq(0.4 \ldots 16) \times 10^{-6}$ или $D_{\sigma} \leq$-(48...64) дБ (рис. 2).

Основной особенностью РЛС с квазинепрерывным излучением является невозможность получения полной пространственной развязки между приемным и передающим трактами. На их входах приемных устройств таких РЛС постоянно присутствует сигнал помехи от передатчика. При этом спектр шумов мешающего сигнала передатчика после его преобразования создает помеху во всем рабочем диапазоне спектроанализатора приемного устройства.

Рассмотрим случай обнаружения малоразмерной цели, находящейся на максимальной дальности, на фоне помех, вносимых шумами сигнала передатчика. Допустимый уровень данной помехи также определим из условия допустимого снижения вероятности правильного обнаружения.

Используя уравнение радиолокации, запишем

$$
N \leq 2 \frac{q_{1}^{2}-q_{2}^{2}}{q_{1}^{2} q_{1}^{1}} \frac{E_{\mathrm{и}} G(\beta, \varepsilon) A(\beta, \varepsilon) S}{\left(4 \pi R_{\text {Ц }}^{2}\right)^{2}},
$$

где: $\mathrm{E}_{И}$ - энергия сигнала за один период зондирования; $G(\beta, \varepsilon)$ - коэффициент усиления передающей антенны; $\mathrm{A}(\beta, \varepsilon)$ - эффективная площадь приемной антенны; $\beta, \varepsilon$ - координаты цели по углу места и азимуту; $S$ - эффективная площадь рассеивания цели; $R_{ц}$ - расстояние до цели.

На вход приемного устройства попадает лишь часть энергии шумов зондирующего сигнала $\mathrm{E}_{\Pi}=\mathrm{E}_{И} \mathrm{~K}_{\mathrm{P}}$, где $\mathrm{K}_{\mathrm{P}}-$ коэффициент развязки между передающим и приемным трактами РЛС. Разделив обе части неравенства на Е пустимый уровень искажений в ЦСС с УМ. Так как в РЛС с квазинепрерывным излучением используется корреляционный метод обработки сигналов с УМ, то отношение $N_{\mathrm{p}} / \mathrm{E}_{\Pi}$ пропорционально максимальному относительному среднеквадратическому значению искажений ВКФ сигнала с УМ

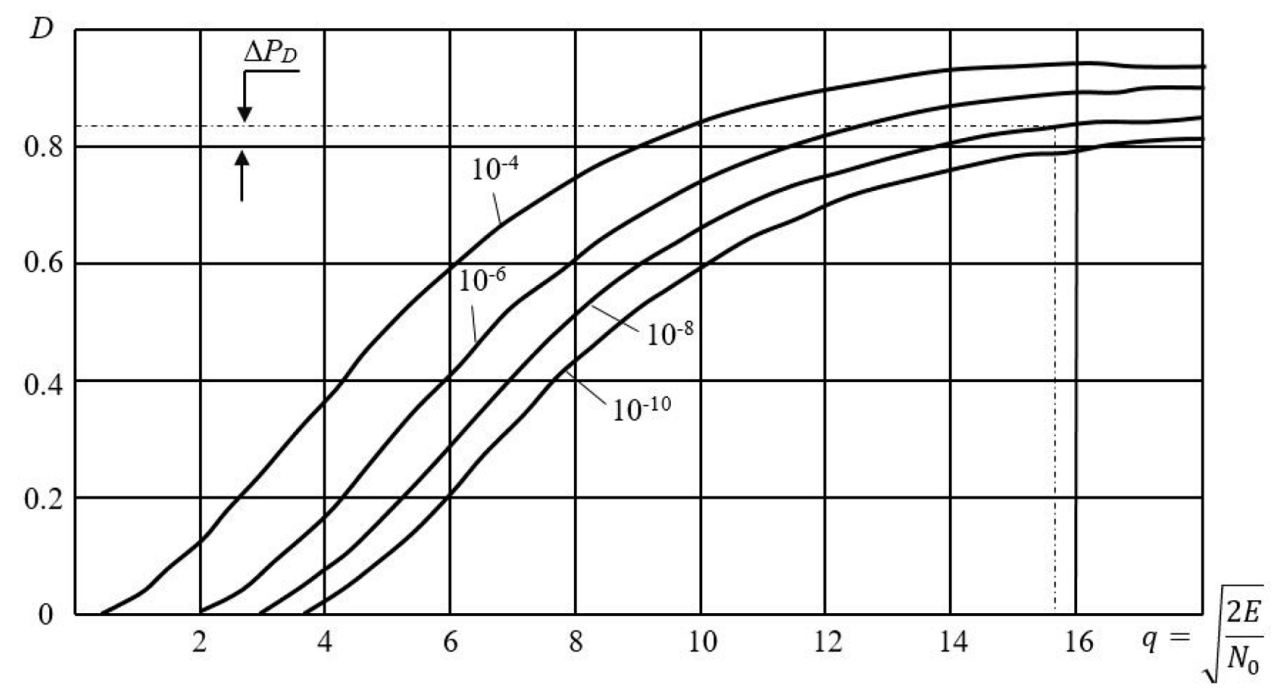

Рис. 2

Fig. 2 


$$
\sigma_{\Delta \chi}^{2} \leq \frac{1}{I} \frac{q_{1}^{2}-q_{2}^{2}}{q_{1}^{2} q_{1}^{1}} \frac{G(\beta, \varepsilon) A(\beta, \varepsilon) S}{K_{\mathrm{p}}\left(4 \pi R_{\mathrm{L}}^{2}\right)^{2}}
$$

Задаваясь значениями $\Delta P_{D}=0.05 \ldots 0.1, P_{D}=0.8 \ldots 0.9, P_{F}=10-8, I=4, G(\beta, \varepsilon)=(3 \ldots 5) \times 10^{4}$, $A(\beta, \varepsilon)=(2 \ldots 4) \times 10^{4} \mathrm{M}^{2}, S=(0.8 . .2) \mathrm{M}^{2}, \mathrm{~K}_{\mathrm{P}}=-(80 \ldots 120)$ дБ, $R_{Ц}=(250 \ldots 400)$ км, получим $\sigma_{\Delta \chi}^{2}=10^{-12} \ldots 10^{-8}$ или $D_{\sigma} \leq-(80 \ldots 120)$ дБ (рис. 2).

Таким образом, в импульсных РЛС максимальное относительное среднеквадратическое значение искажений ВКФ сигнала с УМ, формируемого ЦС, не должно превышать $D_{\sigma} \leq-(51 . .67)$ дБ (рис. 2). В РЛС с квазинепрерывным излучением максимальное относительное среднеквадратическое значение искажений автокорреляционной функции (АКФ) сигнала с УМ, формируемого ЦСС, не должно превышать $D_{\sigma} \leq-(80 . .120)$ дБ (рис. 2).

\section{Рекомендации по выбору количества разрядов квантования фазы, напряжения и частоты дискретизации в ЦСС}

В зависимости от вида ЦСС количество источников детерминированных искажений К различно. При формировании сигнала с УМ в цифровых синтезаторах отсчетов фазы (ЦСОФ) и цифровых синтезаторах отсчетов напряжения (ЦСОН) с (неравномерной дискретизацией) НД осуществляется квантование фазы и его дискретизация. В ЦСОН с равномерной дискретизацией (РД) табличного типа осуществляется квантование амплитуды и дискретизация формируемого сигнала с УМ. Поэтому количество источников детерминированных искажений в этих синтезаторах равно К ии $=2$. В ЦСОН с РД комбинированного типа при формировании сигнала с УМ осуществляется квантование фазы и амплитуды, а также его дискретизация, поэтому $\mathrm{K}_{\text {ии }}=3$. При разработке ЦСС целесообразно, чтобы среднеквадратическая величина каждого источника детерминированных искажений $\sigma_{i}^{2}$, где $I=1,2, \ldots K_{\text {ии }}$, была бы одинаковой. С учетом сказанного выше, используя выражение, определяющее максимальное относительное значение среднеквадратических искажений ВКФ сигналов с УМ, формируемых ЦСС с РД $(R-$ количество отсчетов формируемого сигнала с УМ), для $i$-го источника детерминированных искажений можно записать

$$
\sigma_{i}^{2} \leq \frac{\sigma_{\Delta \chi}^{2}}{K_{\text {ии }}}
$$

В ЦСС ширину рабочего диапазона частот, как правило, выбирают равным четверти тактовой частоты. С учетом выше сказанного относительный среднеквадратический уровень искажений, вносимых каждым источником для импульсных РЛС, составляет $\sigma_{i}^{2}=4 \cdot 10^{-7} \ldots 1,6 \cdot 10^{-5}$ при $K_{\text {ии }}=2$, и $\sigma_{\mathrm{i}}^{2}=2,6 \cdot 10^{-7} \ldots 1 \cdot 10^{-5}$ при $K_{\text {ии }}=3$. Для РЛС с квазинепрерывным излучением $\sigma_{\mathrm{i}}^{2}=2 \cdot 10^{-12} \ldots 2 \cdot 10^{-8}$ при $K_{\text {ии }}=2$, и $\sigma_{\mathrm{i}}^{2}=1,6 \cdot 10^{-12} \ldots 1,6 \cdot 10^{-3}$ при $K_{\text {ии }}=3$.

Количество разрядов квантования фазы для ЦСС с РД можно определить из выражения, где $m$ - количество двоичных разрядов квантования фазы

$$
m \geq \lg \left[\frac{\pi^{2}}{3 R \sigma_{i}^{2}}\right] / 2 \lg 2 .
$$

Количество разрядов квантования напряжения для ЦСС с РД можно получить из выражения, где $\ell$ - количество двоичных разрядов квантования напряжения

$$
-823-
$$




$$
m \geq \lg \left[\frac{2}{3 R \sigma_{i}^{2}}\right] / 2 \lg 2 .
$$

Количество разрядов компенсации временной задержки $q$ и квантования фазы $m$ в многоуровневых ЦСС с НД можно найти из выражения, определяющего максимальное среднеква-

дратическое значение искажений ВКФ $\sigma_{\Delta \chi 2}^{2}=\frac{2 P / R}{(M Q)^{2} R}$ для многоуровневых ЦСС с НД, подставляя в него выражение $M Q=2^{m+q}$,

$$
m+q \geq \lg \left[\frac{4 P}{R^{2} \sigma_{i}^{2}}\right] / 2 \lg 2 .
$$

В двухуровневых ЦСС с НД количество разрядов компенсации временной задержки $q$ находим из выражения, определяющего максимальное среднеквадратическое значение искажений $\sigma_{\Delta \chi 2}^{2}=\frac{2 P / R}{4 Q^{2} R}$, в виде

$$
q \geq \lg \left[\frac{P}{R^{2} \sigma_{i}^{2}}\right] / 2 \lg 2 .
$$

Выбор тактовой частоты ЦСС с РД в общем случае зависит как от ширины спектра сигнала с УМ, так и от его компактности. Численное значение тактовой частоты $\omega_{\text {т }}$ ЦСС с РД можно получить из выражения, характеризующего максимальное относительное среднеквадратическое значение искажений ВКФ, обусловленных дискретизацией, а также квантованием фазы и напряжения сигналов с УМ в ЦС с РД $\sigma_{\Delta \chi_{T}}^{2} \leq\left[\delta_{\varphi, u}^{2}+\delta_{T}^{2}\left(\omega_{\ni}\right) \frac{\omega_{\ni}}{\Delta \omega_{\ni \phi}} \frac{\left(\Delta \omega_{\ni \phi} / \omega_{\ni}+1\right)^{\gamma+1}-1}{2^{\gamma+1}-1}\right]$.

Значение тактовой частоты для линейно-частотно модулированных (ЛЧМ) сигналов, формируемых ЦСОН с РД, можно определить из графика на рис. 3.

Как следует из формул (7) ... (9, б), количество разрядов квантования фазы, напряжения и компенсации временной задержки в ЦСС зависит не только от максимального относительного среднеквадратического значения искажений ВКФ $\sigma_{\mathrm{i}}^{2}$, но и от количества $R$ отсчетов сигнала с УМ. Поэтому непосредственный выбор их можно производить, задаваясь конкретным видом и параметрами сигнала с УМ.

В табл. 1 приведены расчетные данные количества разрядов квантования фазы $m$, напряжения $\ell$ и компенсации временной задержки $q$ при использовании в импульсных РЛС и РЛС с квазинепрерывным излучением ЛЧМ сигнала с девиацией $\Delta f=5$ МГц. Расчеты производились из условия, что эталонная частота ЦСС равна $f_{\ni}=20$ МГц, длительность ЛЧМ сигнала для импульсной РЛС $\mathrm{T}_{\text {и }}=20$ мкс, а полоса пропускания спектроанализатора в РЛС с квазинепрерывным излучением $\Delta f_{\text {СП }}=200$ Гц.

Таким образом, при предъявлении требований к суммарному уровню искажений целесообразно использовать критерий допустимого снижения вероятности правильного обнаружения по сравнению с ее потенциальным значением при фиксированной вероятности ложной тревоги. Исходя из данного критерия в импульсных РЛС максимальное относительное среднеквадратическое значение искажений ВКФ сигнала с УМ, формируемого ЦСС, не должно превы- 


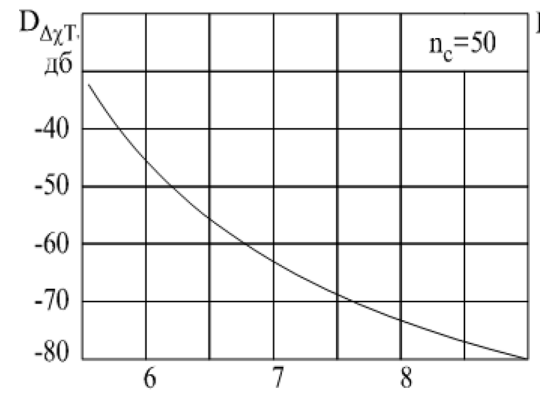

a)

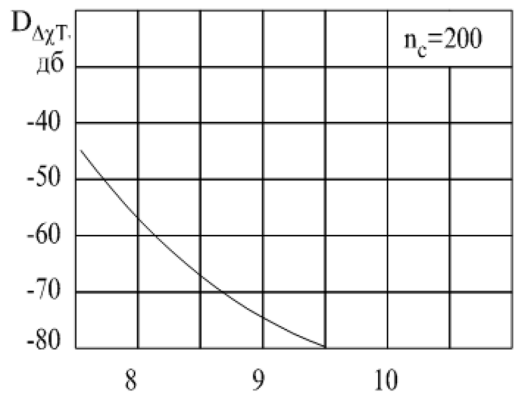

B)

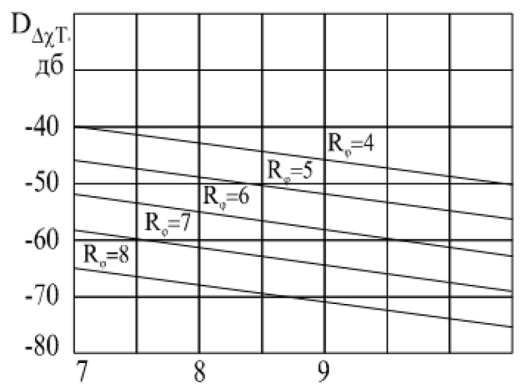

д)

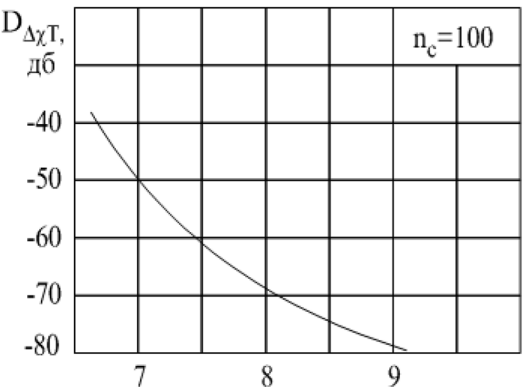

б)

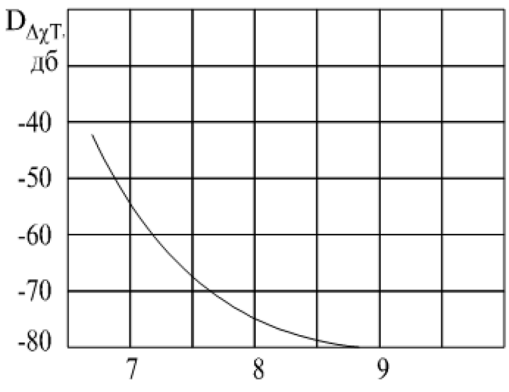

г)

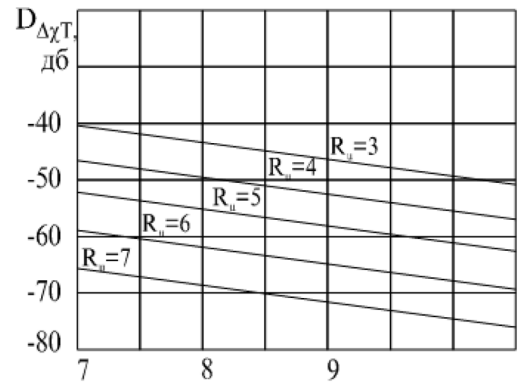

e)

Рис. 3

Fig. 3

Таблица 1

Table 1

\begin{tabular}{|c|c|c|c|c|c|}
\hline \multirow{2}{*}{ Вид РЛС } & & \multicolumn{2}{|c|}{ ЦСОН } & \multicolumn{2}{|c|}{ ЦСОФ } \\
\hline & & с РД & с НД & с РД & с НД \\
\hline \multirow{3}{*}{$\begin{array}{l}\text { Импульсные РЛС } \\
\left(\Delta_{\Delta x}=-(51-67) \text { дБ); }\right. \\
\mathrm{T}_{\text {и }}=20 \text { мкс; } \Delta \mathrm{f}=5 \text { МГц; } \\
\text { fэ = } 20 \text { МГц; } \mathrm{P} / \mathrm{R}=0.5)\end{array}$} & $m$ & $6-9$ & $6-8$ & $6-8$ & $6-8$ \\
\hline & $l$ & $5-8$ & - & - & - \\
\hline & $q$ & - & $4-7$ & - & $4-7$ \\
\hline \multirow{3}{*}{$\begin{array}{l}\text { РЛС с квазинепрерывным излучением } \\
\left(\Delta_{\Delta p}=-(80-120) \text { дБ); }\right. \\
\Delta \mathrm{f}_{\mathrm{C}} \Pi=200 \mathrm{MГц;} \\
\text { Fэ }=20 \text { МГц; } \mathrm{P} / \mathrm{R}=0.5)\end{array}$} & $m$ & $7-14$ & $7-14$ & $7-14$ & $7-14$ \\
\hline & $l$ & $5-12$ & - & - & - \\
\hline & $q$ & - & $5-12$ & - & $5-12$ \\
\hline
\end{tabular}


шать $D_{\delta x} \leq-(51 . .67)$ дБ. В РЛС с квазинепрерывным излучением максимальное относительное среднеквадратическое значение искажений АКФ сигнала с УМ, формируемого ЦСС, не должно превышать $D_{\delta} \leq-(80 \ldots 120)$ дБ.

Количество разрядов квантования фазы, напряжения и компенсации временной задержки в ЦСС зависит не только от максимального относительного среднеквадратического значения искажений ВКФ, но и от количества отсчетов сигнала с УМ. Поэтому первоначально необходимо выбрать эталонную частоту ЦСС, задаваясь видом модуляции и эффективной шириной спектра сигнала с УМ, исходя из ТТХ РЛС.

\section{Список литературы / References}

[1] Кочемасов В., Скок Д., Черкашин А. Цифровые вычислительные синтезаторы - современные решения. Электроника: НТБ, 2014, 2, 150-160. [Kochemasov V., Skok D., Cherkashin A. Digital computing synthesizers - modern solutions. Electronics: STL, 2014, 2, 150-160 (in Russian)]

[2] Кочемасов В., Скок Д., Черкашин А. Цифровые вычислительные синтезаторы - coвременные решения. Ч. 2. Электроника: НТБ, 2014, 4, 152-156. [Kochemasov V., Skok D., Cherkashin A. Digital computing synthesizers - modern solutions. Part 2. Electronics: STL, 2014, 4, 152-156 (in Russian)]

[3] Викторов Д.С., Пластинина Е.В., Гарин Е.Н., Лютиков И.В. Метод компенсации искажений сигналов с угловой модуляцией в цифровых синтезаторах сигналов. Успехи современной радиоэлектроники. М.: Радиотехника, 2017, 12, 86-89. [Viktorov D.S., Plastinina E.V., Garin E.N., Lyutikov I.V. Method of compensating for distortions of signals with angular modulation in digital signal synthesizers. Successes of modern radio electronics. M., Radio engineering, 2017, 12, 86-89 (in Russian)]

[4] Викторов Д.С., Пластинина Е.В. Коррекция нелинейных искажений в цифровых формирователях сигналов. Журнал Сибирского федерального университета. Техника и технологии, 2018, 13(3), 336-346. [Viktorov D.S., Plastinina E.V. Correction of nonlinear distortions in digital signal generators. Journal of the Siberian Federal University. Engineering \& Technologies, 2018, 11(3), 336-346 (in Russian)]

[5] Викторов Д.С., Пластинина Е.В. Методы уменьшения неравномерности передаточной характеристики формирователя аналоговых отсчетов цифровых синтезаторов сигналов с равномерной дискретизацией. Журнал Сибирского федерального университета. Техника и технологии, 2019, 12(6), 630-640. [Viktorov D.S., Plastinina E.V. Methods to reduce unevenness of transmission characteristic of analog samples generator of digital signal synthesizers with uniform sampling. Journal of the Siberian Federal University. Engineering \& Technologies, 2019, 12(6), 630-640 (in Russian)]

[6] Викторов Д.С., Пластинина Е.В. Методы уменьшения модуляционных искажений в трактах формирования и приемно-передающих трактах РЛС. Журнал Сибирского федерального университета. Техника и технологии, 2018, 11(7), 451-461. [Viktorov D.S., Plastinina E.V. Methods for reducing modulation distortions in the formation paths and receiving-transmitting paths of radar stations. Journal of the Siberian Federal University. Engineering \& Technologies, 2018, 11(7), 451-461 (in Russian)]

[7] Викторов Д.С., Пластинина Е.В. Методика определения относительной среднеквадратической оценки искажения спектра сигнала с угловой модуляцией в цифровых синтеза- 
торах с равномерной дискретизацией. Журнал Сибирского федерального университета. Техника и технологии, 2020, 13(3), 259-271. [Viktorov D.S., Plastinina E.V. Method of determining relative RMS estimation of distortion of signal spectrum with angular modulation in digital synthesizers with uniform sampling. Journal of the Siberian Federal University. Engineering \& Technologies, 2020, 13(3), 259-271 (in Russian)]

[8] Сосулин Ю.Г., Костров В.В., Паршин Ю.Н. Оценочно-корреляционная обработка сигналов и компенсация помех. М.: Радиотехника, 2014. 632 с. [Sosulin Yu.G., Kostrov V.V., Parshin Yu.N. Reference correlation alarm and interference compensation. M.: Radio engineering, 2014. 632 p. (in Russian)]

[9] Курилов И.А., Рудаков А.М., Харчук С.М., Романов Д.Н. Математическое моделирование автокомпенсации фазовых помех на выходе ЦАП прямого цифрового синтезатора частот. Радиотехнические и 144 телекоммуникационные системы, 2013, 2(10), 19-25. [Kurilov I.A., Rudakov A.M., Kharchuk S.M., Romanov D.N. Mathematical modeling of automatic compensation of phase interference at the output of the DAC direct digital frequency synthesizer. Radio engineering and 144 telecommunication systems, 2013, 2(10), 19-25 (in Russian)]

[10] Васильев Г.С., Курилов И.А., Харчук С.М. Моделирование нелинейного автокомпенсатора фазовых помех ЦАП прямого цифрового синтезатора частот. Радиотехнические и телекоммуникационные системы, 2014, 2, 30-38. [Vasiliev G.S., Kurilov I.A., Kharchuk S.M. Modeling of a non-linear automatic compensator of phase interference of a digital-analog converter of a direct digital frequency synthesizer. Radio engineering and telecommunication systems, 2014, 2, 30-38 (in Russian)]

[11] Гомозов В.И. Требования к параметрам одиночных и составных ЛЧМ сигналов и построение их унифицированных возбудителей для РЛС с когерентными последовательностями импульсов. Военная радиоэлектроника, 1979, 4, 3-18. [Homozov V.I. Requirements to parameters of single and composite linearly frequency modulated signals and construction of their unified exciters for radar stations with coherent pulse sequences. Military Radio Electronics, 1979, 4, 3-18 (in Russian)]

[12] Гомозов В.И. Формирование сложных радиолокационных СВЧ-сигналов $c$ высокой скоростью угловой модуляции. Дисс. д-ра техн.наук. М., 1982. 485 с. [Homozov V.I. Formation of complex radar ultrahigh-frequency signals with high rate of angular modulation. Dr. tech. sci. diss. M., 1982. 485 p. (in Russian)]

[13] Макарычев Е.М. Оценка влияния нелинейных искажений цифрового и аналогового тракта DDS на спектры гетеродинных сигналов в области доплеровских отстроек. Радиотехника, 2015, 4, 105-111. [Makarychev E.M. Evaluation of the effect of non-linear distortions of the digital and analog DDS path on the spectra of heterodyne signals in the area of Doppler rebuilds. Radio engineering, 2015, 4, 105-111 (in Russian)]

[14] Васильев Г.С., Суржик Д.И., Курилов И.А., Харчук С.М., Романов Д.Н. Автоматический компенсатор фазовых помех на выходе цифро-аналогового преобразователя синтезатора частот. Патент на полезную модель № 2015130455 от 22.07.2015. [Vasiliev G.S., Surzhik D.I., Kurilov I.A., Harchuk S.M., Romanov D.N. Automatic compensator of phase interference at the output of a digital-analog frequency synthesizer converter. Patent for a useful model no. 2015130455, 22.07.2015 (in Russian)] 
[15] Зайцев А.Л. Цифровые синтезаторы сигналов с частотной модуляцией и их использование при радиофизических исследованиях планет. Дисс. канд. техн. наук. М.: ИРЭ, 1982. 352 с. [Zaitsev A.L. Digital synthesizers of signals with frequency modulation and their use in radiophysical studies of planets. Dissertation Ph. D. M., IRE Publ., 1982. 352 p. (in Russian)] 